\title{
Quantifying How Staining Methods Bias Measurements of Neuron Morphologies
}

\author{
Roozbeh Farhoodi ${ }^{1 * t}$, Benjamin James Lansdell ${ }^{2 \dagger}$ and Konrad Paul Kording ${ }^{2,3}$ \\ ${ }^{1}$ Department of Mathematics, Sharif University of Technology, Tehran, Iran, ${ }^{2}$ Department of Bioengineering, University of \\ Pennsylvania, Philadelphia, PA, United States, ${ }^{3}$ Department of Neuroscience, University of Pennsylvania, Philadelphia, PA, \\ United States
}

OPEN ACCESS

Edited by:

Arjen van Ooyen,

VU University Amsterdam,

Netherlands

Reviewed by:

Guy Elston,

University of the Sunshine Coast,

Australia

Adhil Bhagwandin,

University of Cape Town, South Africa

Hans-Gert Bernstein,

University in Magdeburg, Germany

*Correspondence:

Roozbeh Farhoodi

roozbehfarhoodi@gmail.com

tThese authors share first authorship

Received: 01 September 2018 Accepted: 25 April 2019

Published: 21 May 2019

Citation:

Farhoodi R, Lansdell BJ and Kording KP (2019) Quantifying How Staining Methods Bias Measurements of Neuron Morphologies. Front. Neuroinform. 13:36. doi: 10.3389/fninf.2019.00036
The process through which neurons are labeled is a key methodological choice in measuring neuron morphology. However, little is known about how this choice may bias measurements. To quantify this bias we compare the extracted morphology of neurons collected from the same rodent species, experimental condition, gender distribution, age distribution, brain region and putative cell type, but obtained with 19 distinct staining methods. We found strong biases on measured features of morphology. These were largest in features related to the coverage of the dendritic tree (e.g., the total dendritic tree length). Understanding measurement biases is crucial for interpreting morphological data.

Keywords: dendritic morphology, staining method, rodent neuroanatomy, neuroinformatics, golgi method, immunostaining, fluorescence microscopy

\section{INTRODUCTION}

There are many techniques through which neuron morphologies may be imaged. These techniques can be classified based on two factors: how they target neurons for imaging, and how the axons and dendrites of the probed neurons are labeled so they are visible for imaging. Neurons may be targeted through their propensity to take up heavy metals or genetic markers, through immunohistochemistry or by direct injection (Elston et al., 1999; Jacobs et al., 2001; Travis et al., 2005; Donohue and Ascoli, 2011; Parekh and Ascoli, 2013, 2015; Carter and Shieh, 2015). Neurons may be labeled using a variety of heavy metals, fluorescent or chromogenic labels are used to allow imaging. We may expect that both targeting and labeling aspects will introduce biases upon the resulting reconstructions of morphology.

Staining with heavy metals remains one of the key imaging techniques. Golgi staining is the oldest such method. In Golgi staining, silver nitrate is introduced to fixed tissue, and the metal is taken up by a sub-population of neurons through a mechanism that remains largely uncharacterized. Neurons are stained in their entirety and then imaged with light microscopy (Koyama, 2013). The method can be subdivided into Rapid Golgi, Golgi-Kopsch, and Golgi-Cox (Koyama, 2013) and each version labels a subset of neurons. Other heavy metals such as osmium and lead can instead be used for dense labeling, which is popular for EM data (Watson, 1958; Tapia et al., 2012). Or, alternatively, lipophilic dyes such as DiOlistics can be introduced ballistically to neurons and allow for Golgi-like staining (Staffend and Meisel, 2011). Since the biological mechanism of heavy staining method is mainly unknown, the extract morphology may be subject to selection bias (Staffend and Meisel, 2011). Because it is relatively simple to perform compared to other methods, heavy metals are a popular staining method for fixed tissues. 
A more recent approach is to target neurons through genetic markers. Fluorescent proteins such as green fluorescent protein (GFP), red fluorescent protein (RFP), and yellow fluorescent protein (YFP) can be introduced transgenically to be expressed in neurons, and then imaged through fluorescent microscopy to reveal morphology (Marshall et al., 1995). The use of fluorescent proteins may be limited to animals for which good genetic tools exist. Fluorescent proteins are introduced under the control of promoter regions that are active for known cell-type markers. They target specific populations of neurons, which may differ from those neurons targeted by other methods. The size of these potential selection biases again remains relatively uncharacterized. Fluorescent techniques are popular because they readily integrate both into genetic and physiological approaches.

Immunostaining has advanced to be a leading staining technique. Immunostaining uses antibodies to target neuronal molecular markers, which can be labeled with fluorescent or chromogenic tags for imaging (Chen et al., 2010; Tanapat, 2013). A common approach relies on biotin variants, such as biocytin or neurobiotin, being conjugated to an antibody (Swietek et al., 2016). A complex of biocytin and its binding partner, avidin, are tagged with a fluorescent or colored label that can then be imaged. The avidin-biotin complex allows imaging through light, fluorescent, or EM microscopy depending on the label. Common fluorescent dyes used with immunostaining include Alexa Fluor (AF) (Carter and Shieh, 2015). Immunostaining targets neurons based on particular molecular markers which allows a broad range of targets. Immunostaining is particularly popular as it readily integrates into the modern molecular approaches.

Finally, neurons can be directly injected (Vaney, 2002; Elston, 2003). Direct injection allows neurons to be labeled in vivo or in slice samples and later imaged in a fixed preparation, meaning electrophysiology can be related to morphology. It is common to directly inject fluorescent dyes such as Lucifer Yellow (Hanani, 2012) or biotin variants such as biocytin or neurobiotin (Klenowski et al., 2017).

Each method comes with idiosyncrasies and methodological steps that can vary across laboratories. For instance, in immunostaining the antibody concentration, length of incubation time, and accessibility to the antigen all must be balanced to produce a good result (Paavilainen et al., 2010; Carter and Shieh, 2015). All these factors may vary from lab to lab and are a known source of variability. For example, it has been shown that hippocampal CA1 neurons measured in rats housed in different labs are not consistent in terms of their morphometry (Scorcioni et al., 2004). Tripathy et al. (2015) have shown similar biases in electrophysiology (Tripathy et al., 2015; Tebaykin et al., 2017). Understanding the effects of staining is thus crucial for the interpretation of downstream analyses.

Each method also targets different neurons and operates through different biochemical processes such that, even if performed within the same lab, morphology measurements can differ by staining method. For instance, during dehydration it is well-known that incubation with different dyes can affect tissue shrinkage which in turn can affect morphology (Grace and Llinás, 1985). Neurobiotin staining is known to affect both electrophysiology and morphology ( $\mathrm{Xi}$ and $\mathrm{Xu}, 1996$ ). In comparing morphology obtained by Golgi-Cox staining and neurobiotin electroporation, it has been shown that neurobiotin-filling revealed significantly larger dendritic arbors and different spine densities compared to GolgiCox-stained neurons (Klenowski et al., 2017). Despite these known issues, there are few systematic studies that examine the size and nature of these biases across the many methods used to quantify morphology.

Large databases of neuron morphologies (Ascoli, 2006) collect data from many labs, each employing different methods. This allows the comparison of data across distinct staining methods. While many experimental aspects of neuron quantification will differ, the staining method is a central experimental choice. As such, it is important to ask what large databases can tell us about the biases induced by staining methods.

Here we quantify the variation in measured neuron morphology related to the staining or the fluorescent labeling method used, though we will refer to both of these as staining method. We analyze rodent data that has been uploaded by various labs to the public morphology repository neuromorpho.org (Ascoli, 2006). We group them based on the biological attributes and the staining methods. By matching on biological attributes and comparing the morphometry of each group we identify the variation that can be explained by different staining methods.

\section{METHODS}

\subsection{Data Acquisition}

We used dendrite morphologies submitted to neuromorpho.org (version 7.4), a publicly available database of morphology. We performed a careful search of neuromoropho.org to identify populations of neurons that allow for an appropriate study of the effect of staining method. We describe the search criteria used below.

To ensure that dendrites were traced completely, we filtered out neurons in the database whose physical integrity of their dendritic reconstructions was labeled as incomplete. We analyzed neurons that are extracted from a healthy animal, by considering only the neurons whose experimental condition were labeled as control. These steps prevent our analysis from including unwanted effects due to poor reconstruction and experimental condition.

We identified populations of neurons sampled from a specific species, age, gender distribution, region, laminar location obtained with a least two staining methods. To do this we restricted our analysis to the neurons from rat to mouse. We grouped neurons into three age classes: young (more than a month and $<2$ months), young adult (between 2 and 6 months) and adult (more than 6 months). To match the gender distribution, we grouped the neurons into three classes: male, female, and male/female. The latter class was used when labs deposited the equal number of neurons from male and female in the same experiment. We matched the cell types as follows. First, we grouped the neurons into two primary cell types: principal cell and interneurons. Then we grouped 


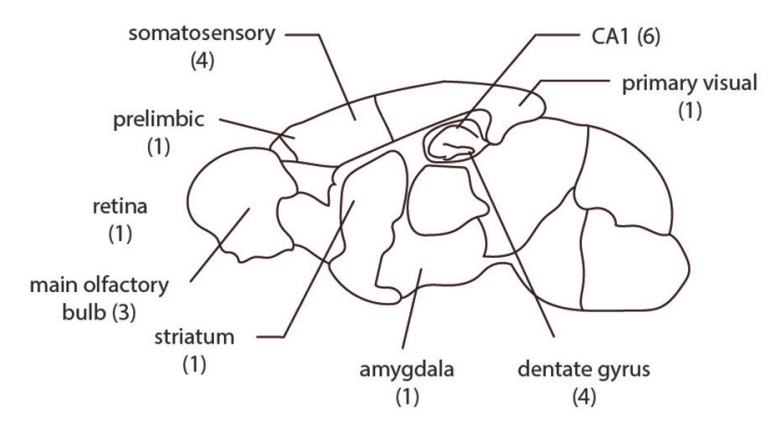

FIGURE 1 | The spatial distribution of the 22 groups analyzed are from multiple brain regions in rodents. The number of comparison groups in each region are listed in parentheses.

each primary cell types into secondary cell type (including pyramidal, granule, mitral, GABAergic). Finally, we grouped them into territory cell type (including Aspiny, spiny, adultborn, newborn) if such information was provided. Similarly, we matched the brain regions as follows. We first grouped them by primary brain regions (neocortex, cerebellum, hippocampus, main olfactory bulb, retina, amygdala, brainstem, entorhinal cortex, spinal cord, protocerebrum). Then we grouped each primary brain regions into secondary brain regions (including primary somatosensory, somatosensory, primary visual, CA1, CA, CA3, dentate gyrus, striatum, anterior cingulate, prelimbic, thalamus, hypothalamus, basolateral amygdala). Then, if each region has a laminar structure (for example somatosensory cortex), we grouped the neurons by their layer [laminar structure contains six distinctive layers (1-6) and three shared layers $(2-3,3-$ 4, 5-6)]. Similarly if the regions had sagittal structure (left, right), or coronal structure (occipital, medial, prefrontal, frontal) or ventral/dorsal structure then we grouped them accordingly. Brain region definitions and nomenclature are taken from the Allen Institute for Brain Science mouse brain atlas, for both the mouse and rat data (Jones et al., 2009). We use it to normalize neuron assignment at the coarse layer (e.g., $C A 1=$ subregion of hippocampus), as the fine structure of the neuron locations is typically not reported. We omitted neurons for which at least one of the above labels was not reported in the database. Using these criteria we grouped the neurons into classes.

If there were at least two different staining methods in a matched group (same brain region, gender distribution, age, species, cell-type) and each staining method has at least five samples in the set, the group was chosen for comparison. In this way we identified 22 matched sets of neurons sourced from more than 60 papers (Figure 1, Tables 1, 2).

In these 22 matched groups of neurons are 19 distinct staining methods, labeled by neuromorpho.org. We grouped these into three types: staining with heavy metals, genetic markers, and immunostaining and direct injection. In the heavy metals group: Golgi, and Golgi-Cox. In the genetic markers group: green fluorescent protein, red fluorescent protein, enhanced green fluorescent protein, and Tag red fluorescent protein. In the immunostaining group: immunostaining, horseradish peroxidase, neurobiotin, biocytin, biocytin \& betaIV-spectrin, Alexa Fluor 488, Alexa 647-dextrane, Alexa Fluor 594, OGB-1, biotinylated dextran amine, lucifer yellow, green fluorescent protein, Alexa Fluor 488, immunostaining, green fluorescent protein, immunostaining.

\subsection{Morphological Features}

To compare neuron morphologies we need to quantify them. The morphology of a neuron is described by a set of points each with a coordinate, diameter and index of its parent point. And a set of edges connecting parent points to their children (Stockley et al., 1993). We used six features to measure the effect of staining. Four features are defined in previous publications and are parts of the L-measure (Scorcioni et al., 2008). Two features are unique to this paper. We classed each feature as either global or local.

Three global features are used. First, the number of branching points in the neuron, or how many times the morphology branched. This feature is defined previously in L-measure. Second, the total length of the dendritic tree. This feature is defined previously in L-measure. Third, the global angle. This measures the angle between the dendritic segment and the vector pointing toward the soma. It provides a measure of how much dendrites point away from the soma (Figure 2). This feature is defined in this paper for the first time.

Three local features are used. First, the branching angle. This is the angle between two edges that branch from a common parent. This feature is defined previously in L-measure. Second, the length of segments. This is the length of dendrite between two consecutive branching nodes. This feature is defined previously in L-measure. Third, the local angle. The local angle measures the angles between the vector connecting the node to its parent and the vector connecting the node to its child. We only consider nodes that have one child. This measures how straight the neurites of the neurons are. This feature is defined for the first time.

For features that are measured per dendrite segment (e.g., branching angle, global angle, local angle), data are pooled over all neurons in the group. In order to avoid artifacts due to software reconstruction of the neuron, we resampled the morphology such that the distance between each consecutive node was equal. To do this we preserved the terminals and branching nodes and selected one node every 10 micrometers (but we suppress the last point if it is within 10 micrometers of the terminal or branch node). This way we obtain a normalized representation that can be compared.

\subsection{Statistical Testing}

We tested for an effect of the staining method on each morphological feature, above effects explained through biological attributes. Our morphological features are generally continuous valued, while neuron classes are categorical. Further, the morphological features generally follow a non-Gaussian distribution (Figure 2). This requires using non-parametric tests. We thus used the Wilcoxon rank-sum test. That is, for each group $b \in \mathcal{B}$, we tested:

$$
H_{0}(B=b): \mu_{1 / 2}\left(M \mid B=b, S=s_{1}\right)=\mu_{1 / 2}\left(M \mid B=b, S=s_{0}\right),
$$


TABLE 1 | Details of groups used in analysis.

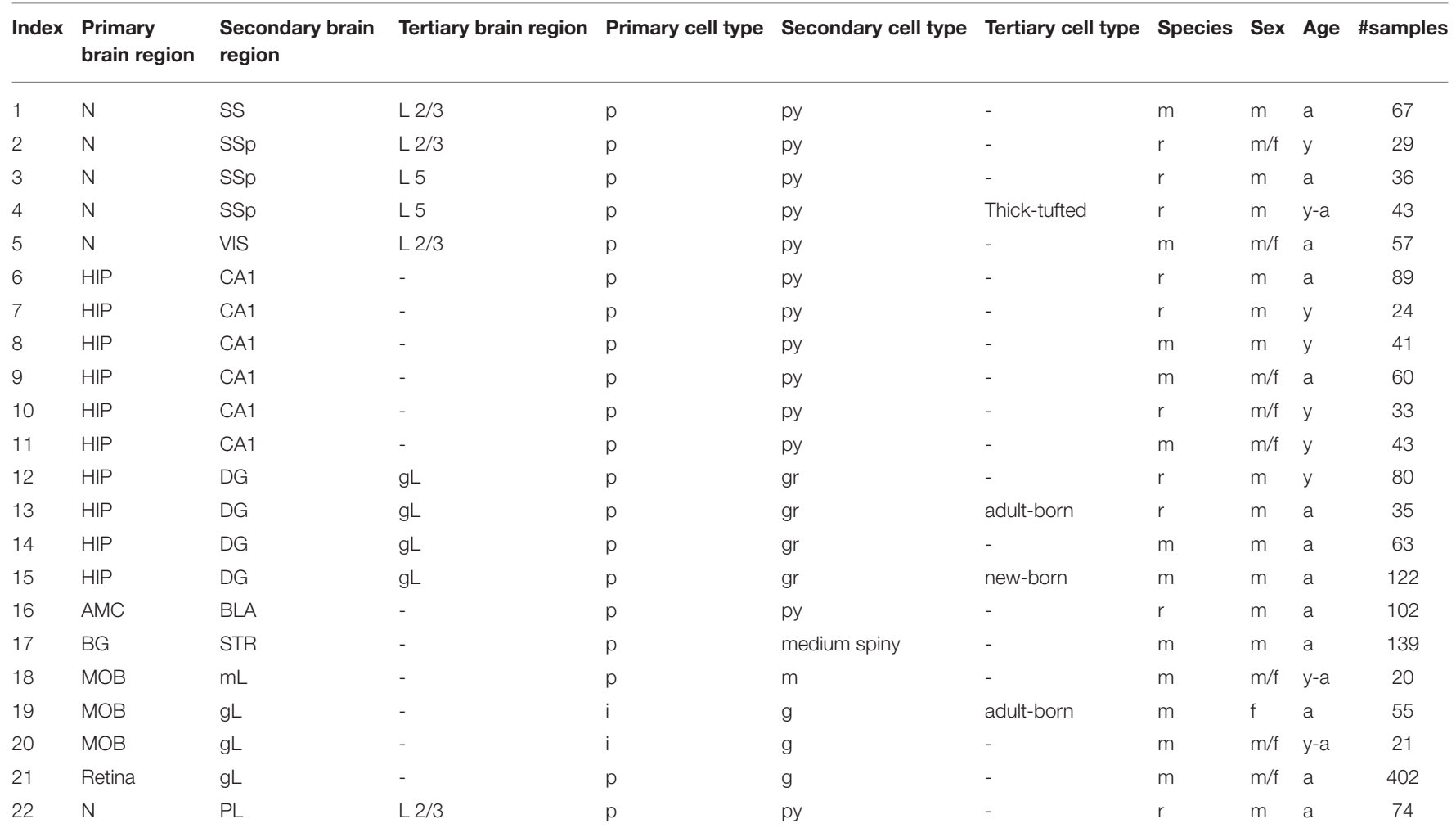

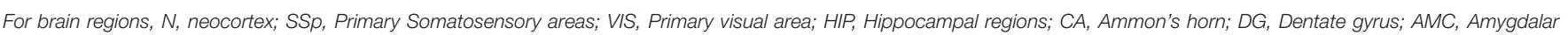

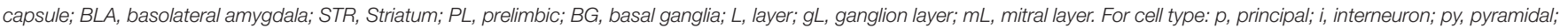
gr, granule; g, ganglion; m, mitral. For species, m, mice; r, rats. For gender distribution, m, male; $f$, female; m/f, male/female. For Age, a, adult, y, young; $y$-a, young-adult.

for all $b \in \mathcal{B}$, where $\mu_{1 / 2}$ represents the median, $S$ the staining method, and $M$ the morphological feature. The hypothesis that no overall effect exists for a given morphological feature is

$$
H_{0}: \cap_{i=1}^{|\mathcal{B}|} H_{0}\left(B=b_{i}\right),
$$

for the $N$ levels in $B$. To correct for multiple testing we used Bonferroni correction.

\subsubsection{Average Effects}

The differences in morphology between staining methods can also be quantified over groups by considering the difference in means:

$$
\beta_{j}=\mathbb{E}\left(\mathbb{E}\left(M_{j} \mid B, S=S_{1}\right)-\mathbb{E}\left(M_{j} \mid B, S=S_{0}\right)\right),
$$

which summarizes the average difference in morphological feature $M_{j}$. This corresponds to the average treatment effect in the causal inference literature (Pearl, 2009), although we can not (and do not) make claims about causality here. A null distribution for each $\beta_{j}$ is generated by repeated permutation of staining label, allowing us to determine significance levels.

\section{RESULTS}

We first asked if neurons obtained by distinct staining methods are distinguishable. Within each group, we compared the distribution of each morphological feature between a pair of staining methods (Figure 2). To do this we tested the hypothesis that the reconstructed morphologies are statistically similar within each group. We observed that, for each pairwise comparison between two staining methods, there is at least one group which shows significant differences in at least one morphological feature (Figure 3, Wilcoxon rank-sum test, $p<$ 0.05 , corrected). In fact, for most of the pairwise comparisons between staining methods we observed a large proportion of highly significant differences (Figure 3, Wilcoxon rank-sum test, $p<0.001$, corrected). This suggests that morphologies obtained by different staining methods seldom agree with each other.

We wondered if the biases in neuronal morphology between staining methods of the same type (e.g., Golgi vs. Golgi-Cox) were less than the biases in morphology between methods of a different type (e.g., Golgi vs. GFP). In fact comparisons between staining methods of the same class showed just as high a proportion of statistically significant differences as comparisons between methods of a different class: in both withinclass and between-class comparisons $90 \%$ of tests performed were statistically significant (Figure 3). Thus, even morphologies obtained by similar methods can show strong biases due to experimental choices.

Given this preponderance of variability related to the staining method, we sought to understand which morphological features 


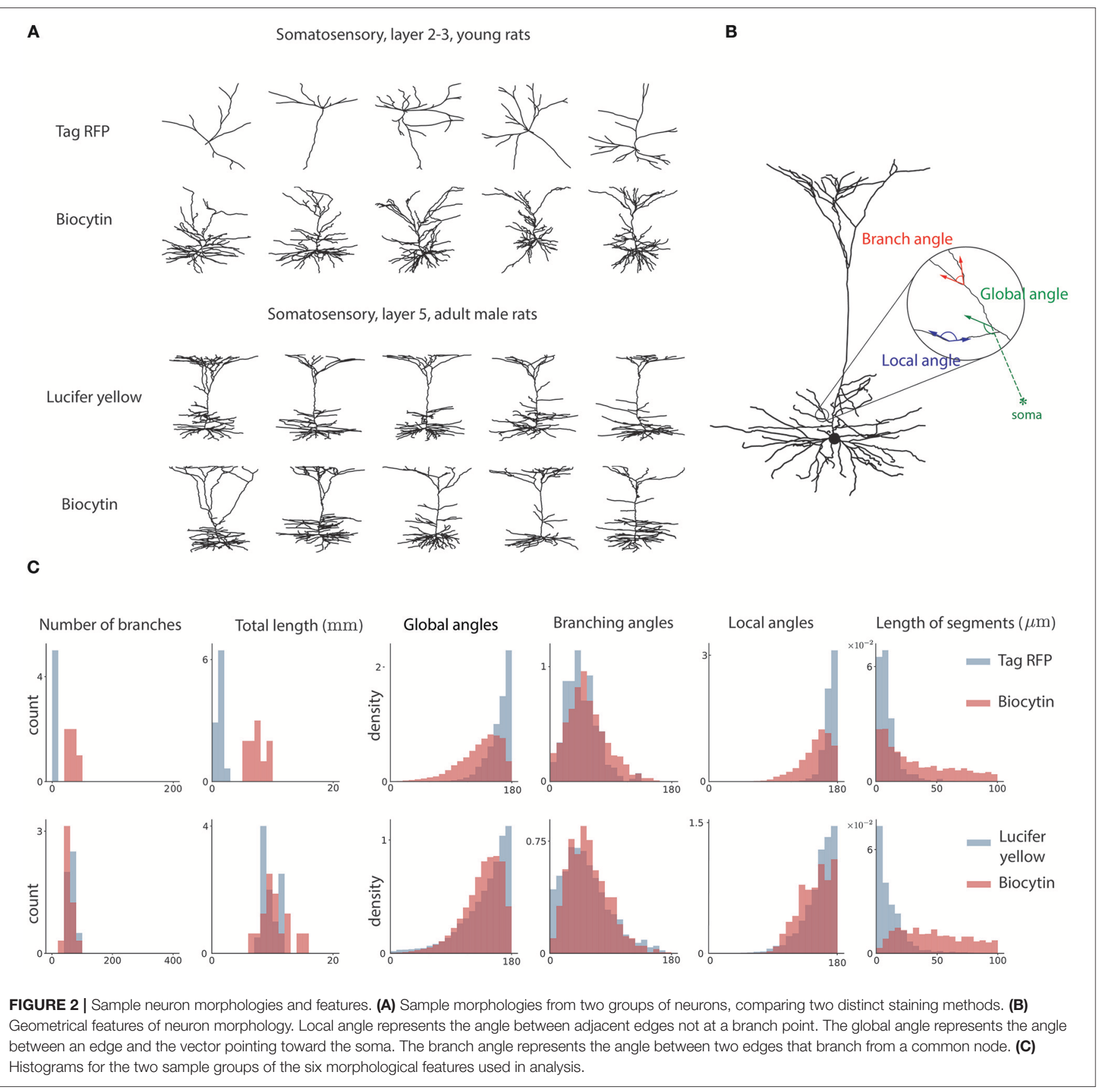

in particular had the strongest biases. In order to examine this we computed the average difference in each feature between each pair of staining methods, averaged over all groups for that comparison. This analysis shows that in general the total length, number of branches, and the length of segments show the strongest biases related to the staining method (Figure 4). Using a permutation test to determine the statistical significance of the average effect, we observed that $76 \%$ of average effects within these three features were significant. While features related to angles of the dendritic tree show weaker effects-only $32 \%$ of average effects in these features were significant. This suggests that features related to the coverage of the dendritic tree are most affected by the choice of staining method.

An omission from these analyses is the possible confounding effect that the rodent strain may have on neuron morphology (Rodriguez et al., 1999; Routh et al., 2009). There is less available data that we could use for matching. Nonetheless, we also analyzed the effect of the staining method on data matched also by strain. This resulted in six comparison groups. In this analysis the staining method biases are of similar significance and size in comparison to the analysis presented above (Supplementary Figures 1, 2). Thus, the biases we observe 


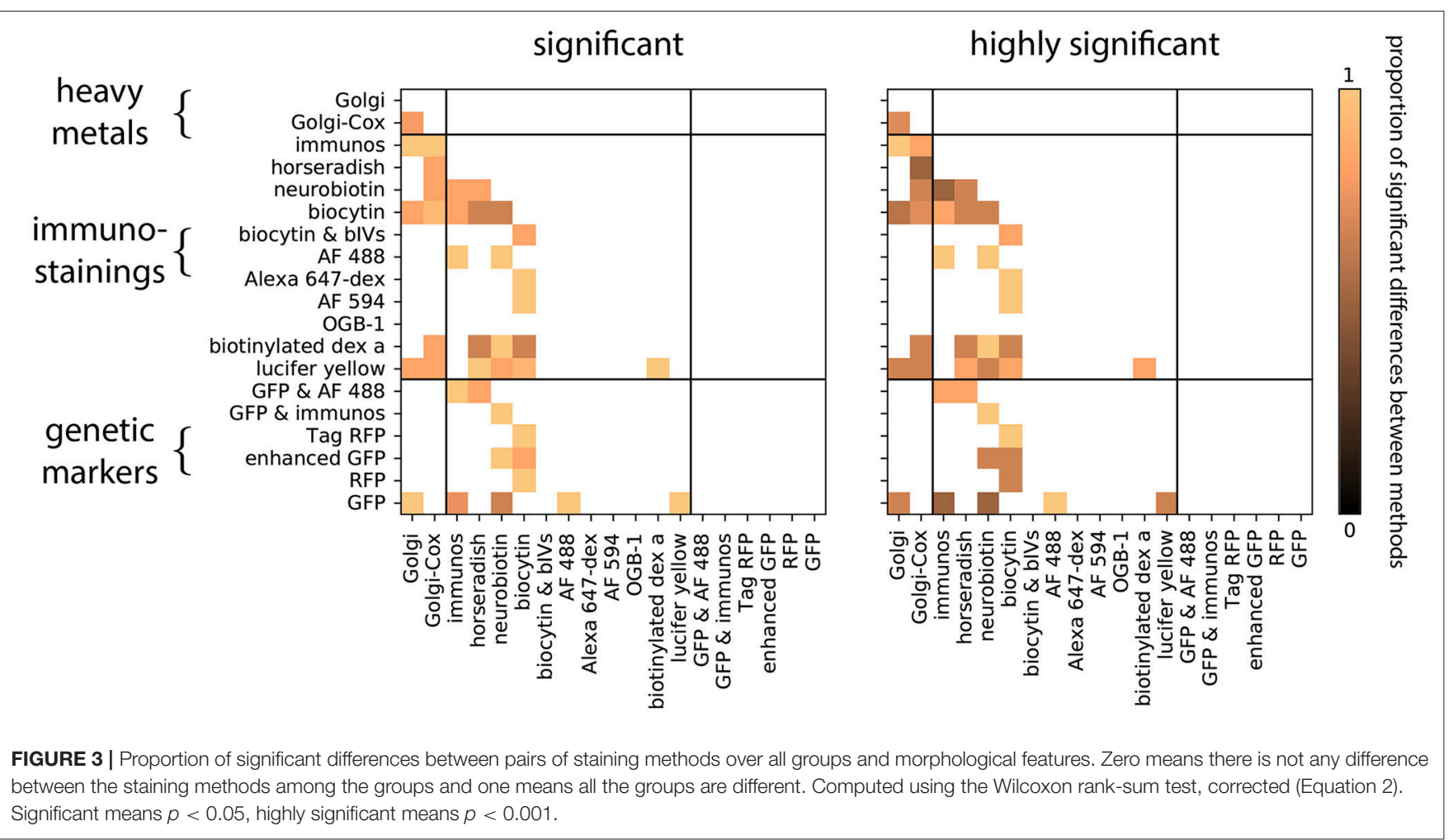

in morphology due to the staining method do not appear to be explained by reported differences in neuron morphology between rodent strains.

A caveat of the available data for our analysis is that it cannot fully separate the effect of the staining method from other laboratory-fixed effects. The ideal dataset to uniquely identify the effect of the staining method would be to have one lab perform the same experiments but with different staining methods. There are a few instances in which such a comparison was made. However, while the data we have thus exhibits a threat to causal validity (Pearl, 2009), we do have sufficient data to estimate how many neurons would be required to estimate such an artifact. To do this we performed a power analysis of the Wilcoxon ranksum test through resampling. For most morphological features, we found that an effect size of $d=0.5$ required 50 neurons/data points to detect the effect with probability $0.9(1-\beta)$, assuming a type I error rate $(\alpha)$ of 0.05 . For features that are defined for each dendrite segment, a single neuron would generally provide this much data. However, to ensure neuron-neuron variability is taken into account, a safer estimate is to assume at least 50 neurons are required. Thus, it seems likely that future versions of neuromorpho.org will soon be able to answer these questions with more precision.

\section{DISCUSSION}

It is important to accurately characterize neuron morphology for a number of reasons. Dendritic morphology determines the computations a neuron can perform, and has a role in circuit function and neurological disease (Agmon-Snir et al., 1998; Elston and Fujita, 2014; Šišková et al., 2014; Yang et al., 2017). Morphology varies by brain region, cell layer, species, and age (Scheibel and Jacobs, 2003; Elston, 2007; Spruston, 2008; Elston and Fujita, 2014). It thus may provide clues as to the function of the region. For instance, Purkinje cells in the cerebellum and pyramidal cells in cortex may provide striking examples of a structure-function relation (Stein and Glickstein, 1992; Körding and König, 2001; Guerguiev et al., 2017). And distinct morphological features affect functional properties differently. For instance, features to do with dendrite diameter may affect electrophysiological properties more than branching angle. Characterizing the morphology specific to brain region, species, etc. is thus important. This is most cleanly identified when the same experimental methodology is used over different brain regions. For instance, Jacobs and Scheibel studied dendritic variation in primate cortical pyramidal cells with the Golgi technique (Scheibel and Jacobs, 2003). Elston and colleagues studied thousands of individually injected cells from multiple cortical areas in a singe hemisphere, replicating the studies in age/sex/hemisphere matched brains within a species and across species (Elston et al., 2001; Elston, 2007). Yet many brain regions and cell types have not been analyzed in this form. Studying variability in morphology by brain region and cell type must typically be performed with data collected from many different methods. Before conclusions can be made, the bias of the methodology must therefore be established.

Here we focused on the correlation of staining methods on measured neuron morphology. We showed a significant 


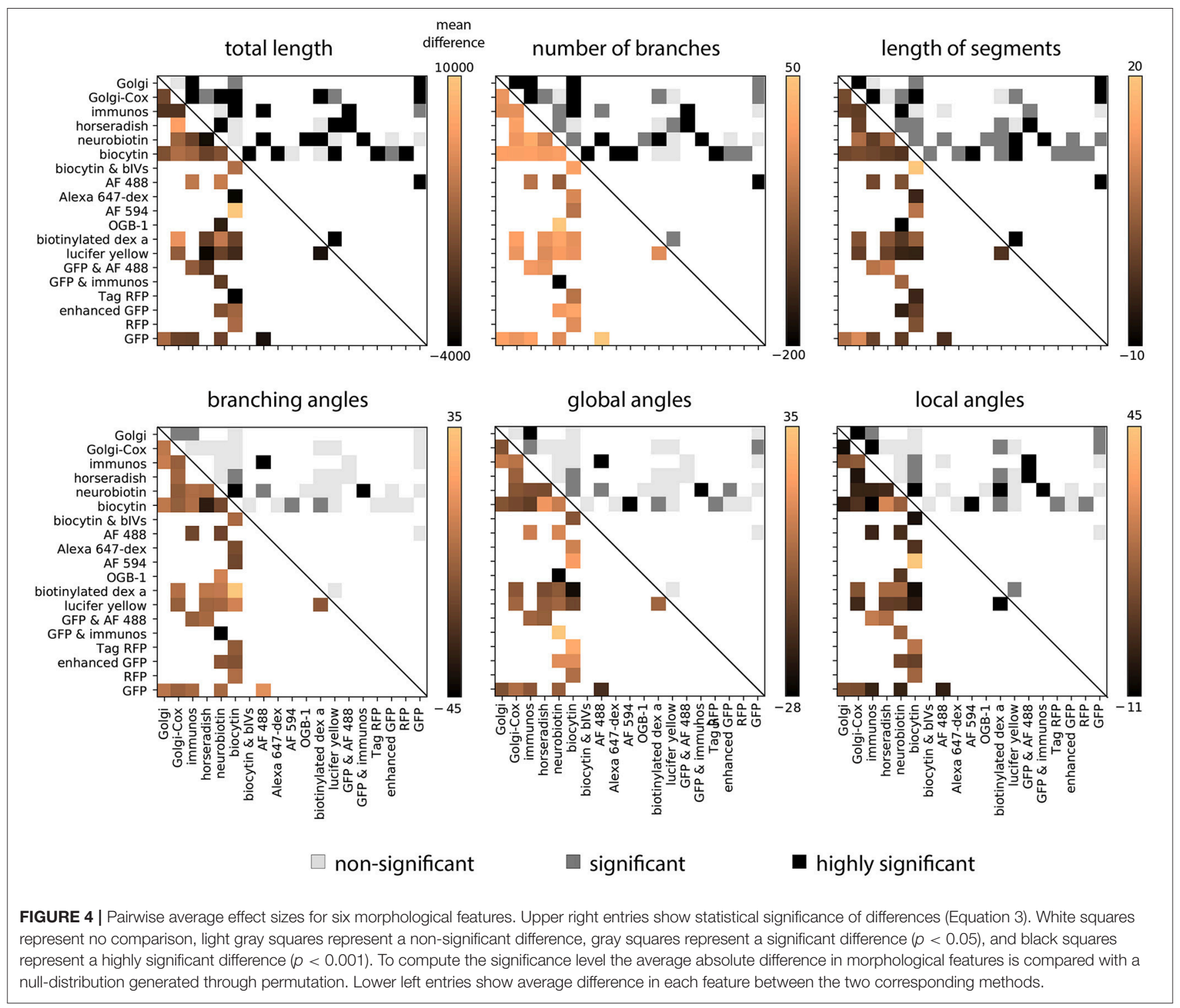

difference between neurons that were extracted from the same region, species, gender distribution, and age but with different staining methods. Although this analysis was focused on the staining method, a similar approach could be taken to study the effect of other methodological details such as the reconstruction software or microscopy method. Understanding the source of these artifacts is necessary for us to have an accurate picture of the variation of neurons in the brain.

There are a number of explanations for biases in morphology related to staining methods. First, there may be procedural differences between laboratories, coming from preferences for particular sub-regions or cell types or other preparation details not reported. Indeed previous studies show this is a large source of variability (Scorcioni et al., 2004). Large differences in morphology can exist within a small region, e.g., visuotopic variation within visual cortex and age (Elston, 2003, 2007; Elston and Fujita, 2014). As such, we may expect significant biases to be related to non-staining related signals.

Second, there are methodological biases related to the $3 \mathrm{D}$ or planar reconstruction of neuron morphology. To produce a $3 \mathrm{D}$ reconstruction of a neuron, we need to fix a direction for the slicing the specimen and choose the thickness of the slices. The staining methods may set a limit on the slice thickness. For instance, when using GFP, neurons are often imaged through confocal microscopy. This sets a bound for the slice thickness, which may affect morphometrics (Rodriguez et al., 2003; Ke et al., 2013). Shrinkage of the neuron during the fixation can also affect measured morphology (Grace and Llinás, 1985). These biases most likely affect the local morphological features, such as angles; the global features, such as total length, are likely less affected. Yet we observed larger biases in global features. This may suggest that biases related to $3 \mathrm{D}$ reconstruction are minimal. 
TABLE 2 | Source references for each comparison group.

\begin{tabular}{|c|c|}
\hline Index & References \\
\hline 1 & $\begin{array}{l}\text { Benavides-Piccione et al., 2005; Alpar et al., 2006; Cohen et al., } \\
2013\end{array}$ \\
\hline 2 & Carrel et al., 2015; Hoffmann et al., 2015 \\
\hline 3 & Kole et al., 2007; Chen et al., 2014 \\
\hline 4 & Kole et al., 2006; Kole, 2011; Hamada et al., 2016 \\
\hline 5 & $\begin{array}{l}\text { Longordo et al., 2013; Jiang et al., 2015; D’Souza et al., 2016; } \\
\text { Vannini et al., } 2016\end{array}$ \\
\hline 6 & $\begin{array}{l}\text { Bannister and Larkman, 1995; Carnevale et al., 1997; Megias } \\
\text { et al., 2001; Kole et al., 2004; Dougherty et al., 2012; Chen et al., } \\
\text { 2014; Malik et al., 2016; Bezchlibnyk et al., } 2017\end{array}$ \\
\hline 7 & $\begin{array}{l}\text { Pyapali et al., 1998; Golding et al., 2005; Scorza et al., 2011; } \\
\text { Groen et al., } 2014\end{array}$ \\
\hline 8 & Suo et al., 2012; Beguin et al., 2013 \\
\hline 9 & Druckmann et al., 2014; Lee et al., 2014; Tyan et al., 2014 \\
\hline 10 & Pyapali et al., 1998; Mulholland et al., 2015 \\
\hline 11 & $\begin{array}{l}\text { Michaelsen et al., 2010; Mendez et al., 2012; Ster et al., 2014; } \\
\text { Tripathy et al., 2015; Zhou et al., 2015; Anstotz et al., 2016; Boillot } \\
\text { et al., } 2016\end{array}$ \\
\hline 12 & Arisi and Garcia-Cairasco, 2007; Beining et al., 2017 \\
\hline 13 & $\begin{array}{l}\text { Rihn and Claiborne, 1990; Carnevale et al., 1997; Beining et al., } \\
2017\end{array}$ \\
\hline 14 & Revest et al., 2009; Winkle et al., 2016 \\
\hline 15 & Carim-Todd et al., 2009; Qin et al., 2014; Platschek et al., 2016 \\
\hline 16 & Bergstrom et al., 2010; Padival et al., 2013; Henckens et al., 2015 \\
\hline 17 & $\begin{array}{l}\text { Martone et al., 2003; Cazorla et al., 2012; Qin et al., 2014; Nato } \\
\text { et al., } 2015\end{array}$ \\
\hline 18 & Fukunaga et al., 2012; Ke et al., 2013 \\
\hline 19 & Belnoue et al., 2016; Sailor et al., 2016 \\
\hline 20 & Burton and Urban, 2015; Quast et al., 2017 \\
\hline 21 & $\begin{array}{l}\text { Chen and Chiao, 2014; Sumbul et al., 2014; Krishnaswamy et al., } \\
\text { 2015; Poria and Dhingra, } 2015\end{array}$ \\
\hline 22 & Soares-Cunha et al., 2014; Henckens et al., 2015 \\
\hline
\end{tabular}

Third, there may exist differences caused by other methodological details that happen to be correlated with the staining method, not because the method goes in hand with the staining method itself, but just by chance (or cultural heritage) in the data we analyzed. For example if the objective type used in the microscopy correlates with different staining methods then that would be a potential confound. However, by performing the same comparison over lab groups and brain regions, we mitigate these confounding effects to some extent, and thus better measure differences that are particular to the staining method. But these other explanations can not be ruled out entirely without more controlled comparisons. This is challenging, even with a large database such as neuromorpho.org. Our power analysis demonstrates how much data would be needed to cleanly address these questions. Ultimately, only a clean experiment with a proper random assignment strategy could produce causal certainty.

Our results are consistent with one mechanistic account by which biases are created from the staining method, and not other potential confounds: different molecule size of agents used in different staining methods target different parts of a neuron. If different staining methods capture different parts of the morphology then we would expect strongest artifacts to be observed between features to do with the amount of dendritic tree described, e.g., total length, dimension, number of branches, etc, and smaller artifacts for more local geometric properties like the branching angles. If, alternatively, the biases observed in morphology were due to staining methods/labs targeting different neuronal subpopulations within a given brain region, then we may expect stronger artifacts to also be observed in local parameters such as branching angle. We do not observe strong artifacts in these parameters. A more careful modeling approach that takes generated neuron morphologies and subsamples them according to a staining model may be able to give a more precise account of the type of biases we may expect due to the staining method, and thus this interpretation could be better tested.

The feature set used here is often used as a basis of cell classification (Vasques et al., 2016). In this regard, our results suggest the need to standardize and carefully characterize these artifacts-after all, such biases could have massive effects on the results of clustering methods used for cell type identification. Alternatively, although some features are affected by different staining method, there are some features that are only weakly affected by the method (Figure 4). It would thus be possible to use features that vary most by cell type and least by staining method as the basis of classification or clustering. This should allow combining our findings with those of previous classification approaches to make the procedures robust to the details of the staining method.

Our analysis tells a cautionary tale about the progressively more popular combination of data sets across labs. Fully characterizing neuronal morphology and its relation to function relies on the generation and analysis of vast amounts of data. Across neuroscience, collaborative efforts across institutions are studying morphology (e.g., Churchland, 2017). Amongst the wealth of datasets available, the need for understanding variability due to the data generation process is important for drawing inferences and analyzing data across disparate sources. This problem is becoming widespread in neuroscience where electrophysiological, molecular, and morphological data are now routinely shared.

\section{DATA AVAILABILITY}

The datasets analyzed for this study can be found in repository neuromorpho (version 7.4) http://neuromorpho.org. The code for performing the analysis is available at https://github.com/ BonsaiNet/Staining-methods-and-morphologies.

\section{AUTHOR CONTRIBUTIONS}

$\mathrm{RF}$ and KK designed the study. RF and BL performed the analysis. $\mathrm{RF}, \mathrm{BL}$, and KK wrote the manuscript. 


\section{ACKNOWLEDGMENTS}

We are grateful to people who helped us to perform this work: David Rolnick for forming the initial formulation; Giorgio Ascoli and Shreejoy Tripathy for reading through the manuscript and providing useful feedback; Samantha Ing-Esteves for valuable ideas and conversation; and the organizers of Neuroinformatics 2018 (http://www.neuroinformatics2018.org/) and CCN 2018 (https://ccneuro.org/2018/default.asp) meetings, where versions of this work were presented. The work of RF was part of

\section{REFERENCES}

Agmon-Snir, H., Carr, C. E., and Rinzel, J. (1998). The role of dendrites in auditory coincidence detection. Nature 393, 268-272. doi: 10.1038/30505

Alpar, A., Ueberham, U., Bruckner, M. K., Seeger, G., Arendt, T., and Gartner, U. (2006). Different dendrite and dendritic spine alterations in basal and apical arbors in mutant human amyloid precursor protein transgenic mice. Brain Res. 1099, 189-198. doi: 10.1016/j.brainres.2006.04.109

Anstotz, M., Huang, H., Marchionni, I., Haumann, I., Maccaferri, G., and Lubke, J. H. (2016). Developmental profile, morphology, and synaptic connectivity of Cajal-Retzius cells in the postnatal mouse hippocampus. Cereb. Cortex 26, 855-872. doi: 10.1093/cercor/bhv271

Arisi, G. M., and Garcia-Cairasco, N. (2007). Doublecortin-positive newly born granule cells of hippocampus have abnormal apical dendritic morphology in the pilocarpine model of temporal lobe epilepsy. Brain Res. 1165, 126-134. doi: 10.1016/j.brainres.2007.06.037

Ascoli, G. A. (2006). Mobilizing the base of neuroscience data: the case of neuronal morphologies. Nat. Rev. Neurosci. 7, 318-324. doi: 10.1038/nrn1885

Bannister, N. J., and Larkman, A. U. (1995). Dendritic morphology of CA1 pyramidal neurones from the rat hippocampus: I. Branching patterns. J. Comp. Neurol. 360, 150-160. doi: 10.1002/cne.903600111

Beguin, S., Crepel, V., Aniksztejn, L., Becq, H., Pelosi, B., Pallesi-Pocachard, E., et al. (2013). An epilepsy-related ARX polyalanine expansion modifies glutamatergic neurons excitability and morphology without affecting GABAergic neurons development. Cereb. Cortex 23, 1484-1494. doi: 10.1093/cercor/bhs138

Beining, M., Jungenitz, T., Radic, T., Deller, T., Cuntz, H., Jedlicka, P., et al. (2017). Adult-born dentate granule cells show a critical period of dendritic reorganization and are distinct from developmentally born cells. Brain Struct. Funct. 222, 1427-1446. doi: 10.1007/s00429-016-1285-y

Belnoue, L., Malvaut, S., Ladevèze, E., Abrous, D. N., and Koehl, M. (2016). Plasticity in the olfactory bulb of the maternal mouse is prevented by gestational stress. Sci. Rep. 6:37615. doi: 10.1038/srep37615

Benavides-Piccione, R., Hamzei-Sichani, F., Ballesteros-Yáñez, I., DeFelipe, J., and Yuste, R. (2005). Dendritic size of pyramidal neurons differs among mouse cortical regions. Cereb. Cortex 16, 990-1001. doi: 10.1093/cercor/bhj041

Bergstrom, H. C., Smith, R. F., Mollinedo, N. S., and McDonald, C. G. (2010). Chronic nicotine exposure produces lateralized, age-dependent dendritic remodeling in the rodent basolateral amygdala. Synapse 64, 754-764. doi: 10.1002/syn.20783

Bezchlibnyk, Y. B., Stone, S. S., Hamani, C., and Lozano, A. M. (2017). High frequency stimulation of the infralimbic cortex induces morphological changes in rat hippocampal neurons. Brain Stimul. 10, 315-323. doi: 10.1016/j.brs.2016.11.013

Boillot, M., Lee, C. Y., Allene, C., Leguern, E., Baulac, S., and Rouach, N. (2016). LGI1 acts presynaptically to regulate excitatory synaptic transmission during early postnatal development. Sci. Rep. 6:21769. doi: 10.1038/srep21769

Burton, S. D., and Urban, N. N. (2015). Rapid feedforward inhibition and asynchronous excitation regulate granule cell activity in the mammalian main olfactory bulb. J. Neurosci. 35, 14103-14122. doi: 10.1523/JNEUROSCI.0746-15.2015

Carim-Todd, L., Bath, K. G., Fulgenzi, G., Yanpallewar, S., Jing, D., Barrick, C. A., et al. (2009). Endogenous truncated TrkB.T1 receptor regulates neuronal his Ph.D. program at Sharif University of Technology under supervision of Dr. Morteza Fotouhi. The authors are grateful for NIH funding (R01MH103910).

\section{SUPPLEMENTARY MATERIAL}

The Supplementary Material for this article can be found online at: https://www.frontiersin.org/articles/10.3389/fninf. 2019.00036/full\#supplementary-material

complexity and TrkB kinase receptor function in vivo. J. Neurosci. 29, 678-685. doi: 10.1523/JNEUROSCI.5060-08.2009

Carnevale, N. T., Tsai, K. Y., Claiborne, B. J., and Brown, T. H. (1997). Comparative electrotonic analysis of three classes of rat hippocampal neurons. J. Neurophysiol. 78, 703-720. doi: 10.1152/jn.1997.78.2.703

Carrel, D., Hernandez, K., Kwon, M., Mau, C., Trivedi, M. P., Brzustowicz, L. M., et al. (2015). Nitric oxide synthase 1 adaptor protein, a protein implicated in schizophrenia, controls radial migration of cortical neurons. Biol. Psychiatry 77, 969-978. doi: 10.1016/j.biopsych.2014.10.016

Carter, M., and Shieh, J. C. (2015). Guide to Research Techniques in Neuroscience. Burlington, MA: Academic Press.

Cazorla, M., Shegda, M., Ramesh, B., Harrison, N. L., and Kellendonk, C. (2012). Striatal D2 receptors regulate dendritic morphology of medium spiny neurons via Kir2 channels. J. Neurosci. 32, 2398-2409. doi: 10.1523/JNEUROSCI.6056-11.2012

Chen, J. R., Wang, B. N., Tseng, G. F., Wang, Y. J., Huang, Y. S., and Wang, T. J. (2014). Morphological changes of cortical pyramidal neurons in hepatic encephalopathy. BMC Neurosci 15:15. doi: 10.1186/1471-2202-15-15

Chen, X., Cho, D.-B., and Yang, P.-C. (2010). Double staining immunohistochemistry. Nat. Am. J. Med. Sci. 2:241. doi: $10.4297 /$ najms.2010.2241

Chen, Y. P., and Chiao, C. C. (2014). Spatial distribution of excitatory synapses on the dendrites of ganglion cells in the mouse retina. PLOS ONE 9:e86159. doi: 10.1371/journal.pone.0086159

Churchland (2017). An international laboratory for systems and computational neuroscience. Neuron 96, 1213-1218. doi: 10.1016/j.neuron.2017.12.013

Cohen, L., Koffman, N., Meiri, H., Yarom, Y., Lampl, I., and Mizrahi, A. (2013). Time-lapse electrical recordings of single neurons from the mouse neocortex. Proc. Natl. Acad. Sci. U.S.A. 110, 5665-5670. doi: 10.1073/pnas. 1214434110

Donohue, D. E. and Ascoli, G. A. (2011). Automated reconstruction of neuronal morphology: an overview. Brain Res. Rev. 67, 94-102. doi: 10.1016/j.brainresrev.2010.11.003

Dougherty, K. A., Islam, T., and Johnston, D. (2012). Intrinsic excitability of CA1 pyramidal neurones from the rat dorsal and ventral hippocampus. J. Physiol. 590, 5707-5722. doi: 10.1113/jphysiol.2012.242693

Druckmann, S., Feng, L., Lee, B., Yook, C., Zhao, T., Magee, J. C., and Kim, J.(2014). Structured synaptic connectivity between hippocampal regions. Neuron 81, 629-640. doi: 10.1016/j.neuron.2013.11.026

D’Souza, R. D., Meier, A. M., Bista, P., Wang, Q., and Burkhalter, A. (2016). Recruitment of inhibition and excitation across mouse visual cortex depends on the hierarchy of interconnecting areas. Elife 5:e19332. doi: 10.7554/eLife.19332

Elston, G. N. (2003). Cortex, cognition and the cell: new insights into the pyramidal neuron and prefrontal function. Cereb. Cortex 13, 1124-1138. doi: 10.1093/cercor/bhg093

Elston, G. N. (2007). Specialization of the neocortical pyramidal cell during primate evolution. Evol. Nervous Syst. 4, 191-242. doi: 10.1016/B0-12-370878-8/00164-6

Elston, G. N., Benavides-Piccione, R., and DeFelipe, J. (2001). The pyramidal cell in cognition: a comparative study in human and monkey. J. Neurosci. 21:RC163. doi: 10.1523/JNEUROSCI.21-17-j0002.2001

Elston, G. N., and Fujita, I. (2014). Pyramidal cell development: postnatal spinogenesis, dendritic growth, axon growth, and electrophysiology. Front. Neuroanat. 8:78. doi: 10.3389/fnana.2014.00078 
Elston, G. N., Tweedale, R., and Rosa, M. G. (1999). Cortical integration in the visual system of the macaque monkey: large-scale morphological differences in the pyramidal neurons in the occipital, parietal and temporal lobes. Proc. R. Soc. Lond. B Biol. Sci. 266, 1367-1374. doi: 10.1098/rspb.1999.0789

Fukunaga, I., Berning, M., Kollo, M., Schmaltz, A., and Schaefer, A. T. (2012). Two distinct channels of olfactory bulb output. Neuron 75, 320-329. doi: 10.1016/j.neuron.2012.05.017

Golding, N. L., Mickus, T. J., Katz, Y., Kath, W. L., and Spruston, N. (2005). Factors mediating powerful voltage attenuation along CA1 pyramidal neuron dendrites. J. Physiol. 568, 69-82. doi: 10.1113/jphysiol.2005.086793

Grace, A. A., and Llinás, R. (1985). Morphological artifacts induced in intracellularly stained neurons by dehydration: circumvention using rapid dimethyl sulfoxide clearing. Neuroscience 16, 461-475. doi: 10.1016/0306-4522(85)90018-1

Groen, M. R., Paulsen, O., Perez-Garci, E., Nevian, T., Wortel, J., Dekker, M. P., et al. (2014). Development of dendritic tonic GABAergic inhibition regulates excitability and plasticity in CA1 pyramidal neurons. J. Neurophysiol. 112, 287-299. doi: 10.1152/jn.00066.2014

Guerguiev, J., Lillicrap, T. P., and Richards, B. A. (2017). Towards deep learning with segregated dendrites. Elife 6:e22901. doi: 10.7554/eLife.22901

Hamada, M. S., Goethals, S., de Vries, S. I., Brette, R., and Kole, M. H. (2016). Covariation of axon initial segment location and dendritic tree normalizes the somatic action potential. Proc. Natl. Acad. Sci. U.S.A. 113, 14841-14846. doi: $10.1073 /$ pnas.1607548113

Hanani, M. (2012). Lucifer yellow - an angel rather than the devil. J. Cell. Mol. Med. 16, 22-31. doi: 10.1111/j.1582-4934.2011.01378.x

Henckens, M. J., van der Marel, K., van der Toorn, A., Pillai, A. G., Fernandez, G., Dijkhuizen, R. M., et al. (2015). Stress-induced alterations in largescale functional networks of the rodent brain. Neuroimage 105, 312-322. doi: 10.1016/j.neuroimage.2014.10.037

Hoffmann, J. H., Meyer, H. S., Schmitt, A. C., Straehle, J., Weitbrecht, T., Sakmann, B., et al. (2015). Synaptic conductance estimates of the connection between local inhibitor interneurons and pyramidal neurons in layer $2 / 3$ of a cortical column. Cereb. Cortex 25, 4415-4429. doi: 10.1093/cercor/bhv039

Jacobs, B., Schall, M., Prather, M., Kapler, E., Driscoll, L., Baca, S., et al. (2001). Regional dendritic and spine variation in human cerebral cortex: a quantitative golgi study. Cereb. Cortex 11, 558-571. doi: 10.1093/cercor/11.6.558

Jiang, X., Shen, S., Cadwell, C. R., Berens, P., Sinz, F., Ecker, A. S., et al. (2015). Principles of connectivity among morphologically defined cell types in adult neocortex. Science 350:aac9462. doi: 10.1126/science. aac 9462

Jones, A. R., Overly, C. C., and Sunkin, S. M. (2009). The allen brain atlas: 5 years and beyond. Nat. Rev. Neurosci. 10:821. doi: 10.1038/nrn2722

Ke, M. T., Fujimoto, S., and Imai, T. (2013). SeeDB: a simple and morphologypreserving optical clearing agent for neuronal circuit reconstruction. Nat. Neurosci. 16, 1154-1161. doi: 10.1038/nn.3447

Klenowski, P., Wright, S., Mu, E., Noakes, P., Lavidis, N., Bartlett, S., et al. (2017). Investigating methodological differences in the assessment of dendritic morphology of basolateral amygdala principal Neurons-A comparison of Golgi-Cox and neurobiotin electroporation techniques. Brain Sci. 7:165. doi: 10.3390/brainsci7120165

Kole, M. H. (2011). First node of Ranvier facilitates high-frequency burst encoding. Neuron 71, 671-682. doi: 10.1016/j.neuron.2011.06.024

Kole, M. H., Brauer, A. U., and Stuart, G. J. (2007). Inherited cortical HCN1 channel loss amplifies dendritic calcium electrogenesis and burst firing in a rat absence epilepsy model. J. Physiol. 578, 507-525. doi: 10.1113/jphysiol.2006.122028

Kole, M. H., Costoli, T., Koolhaas, J. M., and Fuchs, E. (2004). Bidirectional shift in the cornu ammonis 3 pyramidal dendritic organization following brief stress. Neuroscience 125, 337-347. doi: 10.1016/j.neuroscience.2004.02.014

Kole, M. H., Hallermann, S., and Stuart, G. J. (2006). Single Ih channels in pyramidal neuron dendrites: properties, distribution, and impact on action potential output. J. Neurosci. 26, 1677-1687. doi: 10.1523/JNEUROSCI.3664-05.2006

Körding, K. P., and König, P. (2001). Supervised and unsupervised learning with two sites of synaptic integration. J. Comput. Neurosci. 11, 207-215. doi: 10.1023/A:1013776130161

Koyama, Y. (2013). The unending fascination with the golgi method. OA Anatomy 1:24. doi: $10.13172 / 2052-7829-1-3-848$
Krishnaswamy, A., Yamagata, M., Duan, X., Hong, Y. K., and Sanes, J. R. (2015) Sidekick 2 directs formation of a retinal circuit that detects differential motion. Nature 524, 466-470. doi: 10.1038/nature14682

Lee, S. H., Marchionni, I., Bezaire, M., Varga, C., Danielson, N., Lovett-Barron, M., et al. (2014). Parvalbumin-positive basket cells differentiate among hippocampal pyramidal cells. Neuron 82, 1129-1144. doi: 10.1016/j.neuron.2014.03.034

Longordo, F., To, M. S., Ikeda, K., and Stuart, G. J. (2013). Sublinear integration underlies binocular processing in primary visual cortex. Nat. Neurosci. 16, 714-723. doi: 10.1038/nn.3394

Malik, R., Dougherty, K. A., Parikh, K., Byrne, C., and Johnston, D. (2016). Mapping the electrophysiological and morphological properties of CA1 pyramidal neurons along the longitudinal hippocampal axis. Hippocampus 26, 341-361. doi: 10.1002/hipo.22526

Marshall, J., Molloy, R., Moss, G. W., Howe, J. R., and Hughes, T. E. (1995). The jellyfish green fluorescent protein: a new tool for studying ion channel expression and function. Neuron 14, 211-215. doi: 10.1016/0896-6273(95)90279-1

Martone, M. E., Zhang, S., Gupta, A., Qian, X., He, H., Price, D. L., et al. (2003). The cell-centered database: a database for multiscale structural and protein localization data from light and electron microscopy. Neuroinformatics 1, 379-395. doi: 10.1385/NI:1:4:379

Megias, M., Emri, Z., Freund, T. F., and Gulyas, A. I. (2001). Total number and distribution of inhibitory and excitatory synapses on hippocampal CA1 pyramidal cells. Neuroscience 102, 527-540. doi: 10.1016/S0306-4522(00)00496-6

Mendez, P., Pazienti, A., Szabo, G., and Bacci, A. (2012). Direct alteration of a specific inhibitory circuit of the hippocampus by antidepressants. J. Neurosci. 32, 16616-16628. doi: 10.1523/JNEUROSCI.1720-12.2012

Michaelsen, K., Murk, K., Zagrebelsky, M., Dreznjak, A., Jockusch, B. M., Rothkegel, M., et al. (2010). Fine-tuning of neuronal architecture requires two profilin isoforms. Proc. Natl. Acad. Sci. U.S.A. 107, 15780-15785. doi: $10.1073 /$ pnas. 1004406107

Mulholland, P. J., Spencer, K. B., Hu, W., Kroener, S., and Chandler, L. J. (2015) Neuroplasticity of A-type potassium channel complexes induced by chronic alcohol exposure enhances dendritic calcium transients in hippocampus. Psychopharmacology 232, 1995-2006. doi: 10.1007/s00213-014-3835-4

Nato, G., Caramello, A., Trova, S., Avataneo, V., Rolando, C., Taylor, V., et al. (2015). Striatal astrocytes produce neuroblasts in an excitotoxic model of Huntington's disease. Development 142, 840-845. doi: 10.1242/dev.116657

Paavilainen, L., Edvinsson, A., Asplund, A., Hober, S., Kampf, C., Pontén, F., et al. (2010). The impact of tissue fixatives on morphology and antibody-based protein profiling in tissues and cells. J. Histochem. Cytochem. 58, 237-246. doi: 10.1369/jhc.2009.954321

Padival, M. A., Blume, S. R., and Rosenkranz, J. A. (2013). Repeated restraint stress exerts different impact on structure of neurons in the lateral and basal nuclei of the amygdala. Neuroscience 246, 230-242. doi: 10.1016/j.neuroscience.2013.04.061

Parekh, R., and Ascoli, G. A. (2013). Neuronal morphology goes digital: a research hub for cellular and system neuroscience. Neuron 77, 1017-1038. doi: 10.1016/j.neuron.2013.03.008

Parekh, R., and Ascoli, G. A. (2015). Quantitative investigations of axonal and dendritic arbors: development, structure, function, and pathology. Neuroscientist 21, 241-254. doi: 10.1177/1073858414540216

Pearl, J. (2009). Causality. New York, NY: Cambridge University Press. doi: 10.1017/CBO9780511803161

Platschek, S., Cuntz, H., Vuksic, M., Deller, T., and Jedlicka, P. (2016). A general homeostatic principle following lesion induced dendritic remodeling. Acta Neuropathol. Commun. 4:19. doi: 10.1186/s40478-016-0285-8

Poria, D., and Dhingra, N. K. (2015). Spontaneous oscillatory activity in rd1 mouse retina is transferred from $\mathrm{ON}$ pathway to OFF pathway via glycinergic synapse. J. Neurophysiol. 113, 420-425. doi: 10.1152/jn.00702.2014

Pyapali, G. K., Sik, A., Penttonen, M., Buzsaki, G., and Turner, D. A. (1998). Dendritic properties of hippocampal CA1 pyramidal neurons in the rat: intracellular staining in vivo and in vitro. J. Comp. Neurol. 391, 335-352. doi: 10.1002/(SICI)1096-9861(19980216)391:3<335::AID-CNE4>3.0.CO;2-2

Qin, L., Jing, D., Parauda, S., Carmel, J., Ratan, R. R., Lee, F. S., et al. (2014). An adaptive role for BDNF Val66Met polymorphism in motor recovery in chronic stroke. J. Neurosci. 34, 2493-2502. doi: 10.1523/JNEUROSCI.4140-13.2014 
Quast, K. B., Ung, K., Froudarakis, E., Huang, L., Herman, I., Addison, A. P., et al. (2017). Developmental broadening of inhibitory sensory maps. Nat. Neurosci. 20, 189-199. doi: 10.1038/nn.4467

Revest, J. M., Dupret, D., Koehl, M., Funk-Reiter, C., Grosjean, N., Piazza, P. V., et al. (2009). Adult hippocampal neurogenesis is involved in anxiety-related behaviors. Mol. Psychiatry 14, 959-967. doi: 10.1038/mp.2009.15

Rihn, L. L., and Claiborne, B. J. (1990). Dendritic growth and regression in rat dentate granule cells during late postnatal development. Brain Res. Dev. Brain Res. 54, 115-124. doi: 10.1016/0165-3806(90)90071-6

Rodriguez, A., Ehlenberger, D., Kelliher, K., Einstein, M., Henderson, S. C., Morrison, J. H., et al. (2003). Automated reconstruction of three-dimensional neuronal morphology from laser scanning microscopy images. Methods 30, 94-105. doi: 10.1016/S1046-2023(03)00011-2

Rodriguez, I., Feinstein, P., and Mombaerts, P. (1999). Variable patterns of axonal projections of sensory neurons in the mouse vomeronasal system. Cell 97, 199-208. doi: 10.1016/S0092-8674(00)80730-8

Routh, B. N., Johnston, D., Harris, K., and Chitwood, R. A. (2009). Anatomical and electrophysiological comparison of cal pyramidal neurons of the rat and mouse. J. Neurophysiol. 102, 2288-2302. doi: 10.1152/jn.00082.2009

Sailor, K. A., Valley, M. T., Wiechert, M. T., Riecke, H., Sun, G. J., Adams, W., et al. (2016). Persistent structural plasticity optimizes sensory information processing in the olfactory bulb. Neuron 91, 384-396. doi: 10.1016/j.neuron.2016.06.004

Scheibel, A. B., and Jacobs, B. (2003). "Regional dendritic variation in primate cortical pyramidal cells," in Cortical Areas, eds A. Schuez and R. Miller (London: CRC Press), 123-144.

Scorcioni, R., Lazarewicz, M. T., and Ascoli, G. A. (2004). Quantitative morphometry of hippocampal pyramidal cells: differences between anatomical classes and reconstructing laboratories. J. Comp. Neurol. 473, 177-193. doi: $10.1002 / \mathrm{cne} .20067$

Scorcioni, R., Polavaram, S., and Ascoli, G. A. (2008). L-measure: a web-accessible tool for the analysis, comparison and search of digital reconstructions of neuronal morphologies. Nat. Protoc. 3:866. doi: 10.1038/nprot.2008.51

Scorza, C. A., Araujo, B. H., Leite, L. A., Torres, L. B., Otalora, L. F., Oliveira, M. S., et al. (2011). Morphological and electrophysiological properties of pyramidal-like neurons in the stratum oriens of Cornu ammonis 1 and Cornu ammonis 2 area of Proechimys. Neuroscience 177, 252-268. doi: 10.1016/j.neuroscience.2010.12.054

Šišková, Z., Justus, D., Kaneko, H., Friedrichs, D., Henneberg, N., Beutel, T., et al. (2014). Dendritic structural degeneration is functionally linked to cellular hyperexcitability in a mouse model of Alzheimer's disease. Neuron 84, 10231033. doi: 10.1016/j.neuron.2014.10.024

Soares-Cunha, C., Coimbra, B., Borges, S., Carvalho, M. M., Rodrigues, A. J., and Sousa, N. (2014). The motivational drive to natural rewards is modulated by prenatal glucocorticoid exposure. Transl. Psychiatry 4:e397. doi: $10.1038 /$ tp. 2014.45

Spruston, N. (2008). Pyramidal neurons: dendritic structure and synaptic integration. Nat. Rev. Neurosci. 9:206. doi: 10.1038/nrn2286

Staffend, N. A., and Meisel, R. L. (2011). DiOlistic labeling in fixed brain slices: phenotype, morphology, and dendritic spines. Curr. Protoc. Neurosci. Chapter 2:Unit 2.13. doi: 10.1002/0471142301.ns0213s55

Stein, J. F., and Glickstein, M. (1992). Role of the cerebellum in visual guidance of movement. Physiol. Rev. 72, 967-1017. doi: 10.1152/physrev.1992.72.4.967

Ster, J., Steuble, M., Orlando, C., Diep, T. M., Akhmedov, A., Raineteau, O., et al. (2014). Calsyntenin-1 regulates targeting of dendritic NMDA receptors and dendritic spine maturation in CA1 hippocampal pyramidal cells during postnatal development. J. Neurosci. 34, 8716-8727. doi: 10.1523/JNEUROSCI.0144-14.2014

Stockley, E. W., Cole, H. M., Brown, A. D., and Wheal, H. V. (1993). A system for quantitative morphological measurement and electrotonic modelling of neurons: three-dimensional reconstruction. J. Neurosci. Methods 47, 39-51. doi: 10.1016/0165-0270(93)90020-R

Sumbul, U., Song, S., McCulloch, K., Becker, M., Lin, B., Sanes, J. R., et al. (2014). A genetic and computational approach to structurally classify neuronal types. Nat. Commun. 5:3512. doi: 10.1038/ncomms4512
Suo, L., Lu, H., Ying, G., Capecchi, M. R., and Wu, Q. (2012). Protocadherin clusters and cell adhesion kinase regulate dendrite complexity through Rho GTPase. J. Mol. Cell Biol. 4, 362-376. doi: 10.1093/jmcb/mjs034

Swietek, B., Gupta, A., Proddutur, A., and Santhakumar, V. (2016). Immunostaining of biocytin-filled and processed sections for neurochemical markers. J. Vis. Exp. 18, 1-9. doi: 10.3791/54880

Tanapat, P. (2013). Neuronal cell markers. Mater. Methods 3:196. doi: $10.13070 /$ mm.en.3.196

Tapia, J. C., Kasthuri, N., Hayworth, K., Schalek, R., Lichtman, J. W., Smith, S. J., et al. (2012). High contrast en bloc staining of neuronal tissue for field emission scanning electron microscopy. Nat. Protoc. 7:193. doi: 10.1038/nprot.2011.439

Tebaykin, D., Tripathy, S. J., Binnion, N., Li, B., Gerkin, R. C., and Pavlidis, P. (2017). Modeling sources of interlaboratory variability in electrophysiological properties of mammalian neurons. J. Neurophysiol. 119, 1329-1339. doi: 10.1152/jn.00604.2017

Travis, K., Ford, K., and Jacobs, B. (2005). Regional dendritic variation in neonatal human cortex: a quantitative golgi study. Dev. Neurosci. 27, 277-287. doi: $10.1159 / 000086707$

Tripathy, S. J., Burton, S. D., Geramita, M., Gerkin, R. C., and Urban, N. N. (2015). Brain-wide analysis of electrophysiological diversity yields novel categorization of mammalian neuron types. J. Neurophysiol. 113, 3474-3489. doi: $10.1152 /$ jn.00237.2015

Tyan, L., Chamberland, S., Magnin, E., Camire, O., Francavilla, R., David, L. S., et al. (2014). Dendritic inhibition provided by interneuron-specific cells controls the firing rate and timing of the hippocampal feedback inhibitory circuitry. J. Neurosci. 34, 4534-4547. doi: 10.1523/JNEUROSCI.3813-13.2014

Vaney, D. I., Azmitia E. C., DeFelipe, J., Jones, E. G., Rakic, P., Ribak, C.E., et al. (2002). "Retinal neurons: cell types and coupled networks," in Progress in Brain Research, 136, eds E. C. Azmitia, J. DeFelipe, E. G. Jones, P. Rakic, and C. E. Ribak (Elsevier), 239-254. doi: 10.1016/S0079-6123(02)36020-5

Vannini, E., Restani, L., Pietrasanta, M., Panarese, A., Mazzoni, A., Rossetto, O., et al. (2016). Altered sensory processing and dendritic remodeling in hyperexcitable visual cortical networks. Brain Struct. Funct. 221, 2919-2936. doi: 10.1007/s00429-015-1080-1

Vasques, X., Vanel, L., Villette, G., and Cif, L. (2016). Morphological neuron classification using machine learning. Front. Neuroanat. 10:102. doi: 10.3389/fnana.2016.00102

Watson, M. L. (1958). Staining of tissue sections for electron microscopy with heavy metals. J. Cell Biol. 4, 475-478. doi: 10.1083/jcb.4.4.475

Winkle, C. C., Olsen, R. H., Kim, H., Moy, S. S., Song, J., and Gupton, S. L. (2016). Trim9 deletion alters the morphogenesis of developing and adult-born hippocampal neurons and impairs spatial learning and memory. J. Neurosci. 36, 4940-4958. doi: 10.1523/JNEUROSCI.3876-15.2016

Xi, X. Z., and Xu, Z. C. (1996). The effect of neurobiotin on membrane properties and morphology of intracellularly labeled neurons. J. Neurosci. Methods 65, 27-32. doi: 10.1016/0165-0270(95)00140-9

Yang, S.-A., Yoon, J., Kim, K., and Park, Y. (2017). Measurements of morphological and biophysical alterations in individual neuron cells associated with early neurotoxic effects in Parkinson's disease. Cytometry A 91, 510-518. doi: $10.1002 /$ cyto.a. 23110

Zhou, P., Burton, S. D., Snyder, A. C., Smith, M. A., Urban, N. N., and Kass, R. E. (2015). Establishing a statistical link between network oscillations and neural synchrony. PLoS Comput. Biol. 11:e1004549. doi: 10.1371/journal.pcbi.1004549

Conflict of Interest Statement: The authors declare that the research was conducted in the absence of any commercial or financial relationships that could be construed as a potential conflict of interest.

Copyright (C) 2019 Farhoodi, Lansdell and Kording. This is an open-access article distributed under the terms of the Creative Commons Attribution License (CC BY). The use, distribution or reproduction in other forums is permitted, provided the original author(s) and the copyright owner(s) are credited and that the original publication in this journal is cited, in accordance with accepted academic practice. No use, distribution or reproduction is permitted which does not comply with these terms. 